\title{
Association between decreased ovarian reserve and poor oocyte quality
}

\author{
Viviane Margareth Scantamburlo, MD ${ }^{1,2}$, Renate von Linsingen, MD', Lidio Jair Ribas Centa, MD, \\ Kahisa Fontana Dal Toso, MD ${ }^{1}$, Debora Scaraboto, MD1 , Edward Araujo Júnior, PhD ${ }^{3}$, Jaime Kulak Junior, PhD ${ }^{2}$ \\ Department Gynecology and Obstetrics, ${ }^{1}$ Human Reproduction and Andrology Clinic and Laboratory, ${ }^{2}$ Federal University of Paraná (UFPR), Curitiba, \\ PR; ${ }^{3}$ Department of Obstetrics, Paulista School of Medicine-Federal University of São Paulo (EPM-UNIFESP), São Paulo, SP, Brazil
}

\section{Objective}

To analyze the association between oocyte quality and decreased ovarian reserve (DOR) markers in young women undergoing controlled ovarian stimulation (COS).

\section{Methods}

This retrospective study included 49 patients classified as having DOR based on anti-Müllerian hormone (AMH) levels, follicle-stimulating hormone (FSH) levels, or antral follicle counts (AFCs; <10). Images of all obtained oocytes were analyzed, and oocyte quality was classified according to maturity and morphology. The COS protocol utilized gonadotropin (FSH and/or human menopausal gonadotropin [hMG]) doses ranging from 150 to 300 IU/day. The Student's $t$ test or Mann-Whitney test was used to compare the groups. Spearman's coefficients were estimated to verify the correlation between the administered dose of FSH/hMG and the number of mature oocytes. To evaluate the association between patient- and oocyte-related variables, logistic regression models were adjusted.

\section{Results}

Women with DOR classified according to FSH level had more immature oocytes $(P<0.001)$. Women with DOR according to AMH had fewer mature oocytes and increased basal FSH levels $(P<0.001)$. Women with DOR according to AFC had an increased risk of abnormally shaped oocytes $(P=0.035)$.

\section{Conclusion}

This study showed that DOR based on AMH levels, FSH levels, and AFC was associated with poorer quality oocytes in young women who underwent COS.

Keywords: Infertility; Oocyte quality; Ovarian reserve; Controlled ovarian stimulation; Decreased ovarian reserve

\section{Introduction}

The age of a woman who wants to become pregnant is considered an important marker of ovarian reserve and a predictor of success in assisted reproductive technology. Thus, theoretically, younger women ( $<35$ years of age) have a higher chance of becoming pregnant because they have more and higher quality oocytes [1-3].

Women with decreased ovarian reserve (DOR) are those of childbearing age with regular menstrual cycles and decreased response to controlled ovarian stimulation (COS) and/or lower fertility than other women of the same age $[1,3,4]$. DOR is a cause of infertility, and few studies on its prevalence have been conducted in women under the age of 35 years
Received: 2020.06.18. Revised: 2020.11.09. Accepted: 2021.09.03. Corresponding author: Edward Araujo Júnior, $\mathrm{PhD}$

Department of Obstetrics, Paulista School of Medicine-Federal University of São Paulo (EPM-UNIFESP), Rua Belchior de Azevedo, São Paulo-SP 05089-030, Brazil

E-mail: araujojred@terra.com.br

https://orcid.org/0000-0002-6145-2532

Articles published in Obstet Gynecol Sci are open-access, distributed under the terms of the Creative Commons Attribution Non-Commercial License (http://creativecommons. org/licenses/by-nc/3.0/) which permits unrestricted non-commercial use, distribution, and reproduction in any medium, provided the original work is properly cited.

Copyright $\odot 2021$ Korean Society of Obstetrics and Gynecology 


\section{Obstetrics \& Gynecology Science}

Viviane Margareth Scantamburlo, et al. Ovarian reserve and oocyte quality

$[1,4]$. There is no consensus on whether or not oocyte quality is preserved in these women [4]. Consequently, there are doubts about the actual natural pregnancy rate or success rate of assisted reproductive technology in such patients [5]. Tests to estimate ovarian reserve include measuring basal follicle-stimulating hormone (FSH) levels, antral follicle counts (AFCs), and anti-Müllerian hormone (AMH) levels $[3,6,7]$.

The natural decrease in fertility and increased rates of miscarriage owing to aneuploidy that are associated with increasing age in women are established in the literature and have been suggested to result from the poor quality of remaining oocytes [1,5,8-11]. Most of these oocyte abnormalities include modifications in the mitotic spindle constitution mechanism, chromosome alignment errors, increased rate of degeneration, and chromosomal non-disjunction [11-13].

Morphological oocyte evaluation is simple and safe and provides high- fidelity oocyte quality parameters [13]. Oocytes with cytoplasmic changes such as dark central granulation and/or endoplasmic reticulum aggregates have a significantly higher proportion of abnormalities related to the cytoskeleton and number of chromosomes [12].

It is controversial whether variations in oocyte morphology occur due to ovarian hyperstimulation $[13,14]$. The dosedependent adverse effects of FSH as an independent factor have a negative correlation with the probability of live birth [15]. However, it is suspected that the worsening of reproductive outcomes when high FSH doses are used is due to the use of poor quality oocytes and is not a consequence of the medication itself [15].

Few studies have correlated DOR with oocyte quality in young women and investigated whether this relationship impacts on success rates in assisted reproductive technology. Therefore, the objective of this study was to investigate whether an association between DOR (evaluated using three measurements: basal FSH levels, AMH levels, and AFCs) and oocyte quality according to morphology and maturity exists in young women. We also evaluated whether the gonadotropin dose used in COS was associated with an increased risk of oocyte alterations according to the ovarian reserve in these women.

\section{Materials and methods}

This retrospective study analyzed data from patient records of infertile women who underwent COS for in vitro fertilization using their oocytes at a single-assisted reproduction center located in Curitiba, PR, Brazil between May 2012 and June 2016. The study was approved by the Ethics Review Board of the Federal University of Paraná (UFPR; No. CAAE 51021715.9.0000.0096).

Women aged between 18 and 35 years with a history of infertility and with or without previous attempts at assisted reproductive technology were included. The exclusion criteria were as follows: use of hormonal contraceptives or hormone replacement therapy during the 30 days prior to the assessment of ovarian reserve, history of pelvic radiotherapy or chemotherapy, oophorectomy and/or oophoroplasty, endocrinopathies (polycystic ovary syndrome, hyperprolactinemia, and hypopituitarism), autoimmune diseases, or genetic diseases identified by clinicians and confirmed by karyotyping. Patients with incomplete data in their medical records, such as those with missing examination results, were excluded.

Venous blood samples were collected from all included patients on the 2nd or 3rd day of their menstrual cycle to analyze basal FSH levels using chemiluminescence (IMMULITE 2000 XPi, Siemens Healthineers, Erlangen, Germany). AMH levels were measured using an enzyme-linked immunosorbent assay (AMH Gen II, Beckman Coulter, Inc., Brea, CA, USA).

Antral follicles were counted by the same observer using transvaginal ultrasonography. Follicles were considered antral when their size on transvaginal ultrasound was between 2 and $8 \mathrm{~mm}$ on the $2 \mathrm{nd}$ or $3 \mathrm{rd}$ day of menstruation. AFC was the sum of follicles found in both ovaries.

Patients were classified as having normal ovarian reserve (NOR) when the basal FSH levels were $<10 \mathrm{mlU} / \mathrm{mL}$, $\mathrm{AMH}$ levels were $\geq 1.2 \mathrm{ng} / \mathrm{mL}$, or AFC was $\geq 10$. Patients with $\mathrm{FSH}$ levels $>10 \mathrm{mIU} / \mathrm{mL}$, AMH levels $<1.2 \mathrm{ng} / \mathrm{mL}$, or $A F C<10$ were considered to have DOR.

The COS protocol utilized gonadotropins (FSH: follitropin beta, Puregon ${ }^{\circledR}$, Organon, Jersey City, NJ, USA; follitropin alfa, Gonal-F, Merck Serono, Darmstadt, Germany; human urofollitropin, Fostimon ${ }^{\circledR}$, UCB, Brussels, Belgium; and/ or human menopausal gonadotropin [hMG]: menotropin, Menopur ${ }^{\circledast}$, Ferring Pharmaceuticals, Kiel, Germany) and was based on an individually tailored dose ranging from 150 to $300 \mathrm{IU} /$ day according to age, ovarian reserve, and number of oocytes desired. FSH was replaced by hMG at the same dosage in all patients from the 6th day of ovarian stimulation. 


\section{Obstetrics \& Gynecology Science}

Vol. 64, No. 6, 2021

The combined doses of FSH and hMG administered over the entire stimulation period were considered the total dose. The gonadotrophin dose was fixed at the beginning and did not undergo modification during the COS cycle. Only one COS cycle was performed for each patient.

Based on the results of serial ultrasound examinations, ovarian blockage with a GnRH antagonist (ganirelix, Orgalutran ${ }^{\circledast}$, Organon, Ravensburg, Germany) was initiated when more than two follicles of $\geq 14 \mathrm{~mm}$ in size were observed. Urinary human chorionic gonadotropin [hCG] $(5,000$

Table 1. Oocyte quality according to maturity and morphology

\begin{tabular}{l}
\hline Oocyte maturity \\
Mature: oocytes in metaphase II \\
Immature: oocytes in prophase I and metaphase I \\
Oocyte morphology \\
Abnormal shape \\
Abnormal zona pellucida \\
Abnormal perivitelline space (include changes in the polar \\
corpuscle) \\
Abnormal cytoplasm \\
\hline
\end{tabular}

IU; Choriomon ${ }^{\circledR}$, UCB, Brussels, Belgium) or recombinant hCG (Ovidrel, Merck Serono, Darmstadt, Germany) was administered when three or more follicles of $>18 \mathrm{~mm}$ were observed. Under patient sedation, oocytes were retrieved vaginally by ultrasound at 35-36 hours after the luteinizing hormone peak.

Archived images of all harvested oocytes were analyzed using an ECLIPSE Ti Series inverted microscope (Nikon, Tokyo, Japan) at $\times 200$ magnification by the same embryologist. Oocyte quality was classified according to maturity and morphology (Table 1) [16].

Because sperm quality is an important factor for determining fertilization rate and embryonic development and the medical center where the treatments were performed is a reference for treating male-factor infertility, we chose not to assess oocyte fertilization rates and perinatal outcomes.

The student's $t$-test for independent samples (age and dose of FSH/hMG) or non-parametric Mann-Whitney $U$ test (number of mature oocytes and basal FSH levels) was used to compare groups according to $\mathrm{AMH}$ levels ( $<1.2$ or $\geq 1.2 \mathrm{ng} / \mathrm{mL}$ ), AFCs $(<10$ or $\geq 10)$, or basal FSH levels ( $<10$ or $\geq 10 \mathrm{mlU} / \mathrm{mL}$ ). To analyze the correlation between the administered FSH/

Table 2. Characteristics of woman participants $(n=49)$

\begin{tabular}{lcc}
\hline Variable & Mean \pm standard deviation & Median (minimum-maximum) \\
\hline Age $(\mathrm{yr})$ & $31.1 \pm 3.3$ & $31(23-35)$ \\
Spouse age $(\mathrm{yr})$ & $34.2 \pm 5.7$ & $34(24-58)$ \\
Volume of right ovarian at US $\left(\mathrm{cm}^{3}\right)$ & $6.1 \pm 2.9$ & $5.4(1.8-13.9)$ \\
Volume of left ovarian at US $\left(\mathrm{cm}^{3}\right)$ & $7.9 \pm 8.8$ & $6.2(1.1-47.7)$ \\
AFC & $11.7 \pm 6.7$ & $10(2-27)$ \\
Estradiol $(\mathrm{ng} / \mathrm{mL})$ & $55.3 \pm 38.4$ & $44.6(19.0-241.0)$ \\
FSH $(\mathrm{mlU} / \mathrm{mL})$ & $7.4 \pm 2.7$ & $6.5(3.6-17.0)$ \\
LH (mIU/mL) & $5.2 \pm 2.2$ & $4.9(1.6-11.1)$ \\
AMH $(\mathrm{ng} / \mathrm{mL})$ & $1.9 \pm 1.6$ & $1.4(0.1-6.6)$ \\
Height $(\mathrm{m})$ & $1.7 \pm 0.0$ & $1.7(1.6-1.8)$ \\
Weight $(\mathrm{kg})$ & $64.6 \pm 8.2$ & $63.7(51.0-89.0)$ \\
BMI $\left(\mathrm{kg} / \mathrm{m}^{2}\right)$ & $23.2 \pm 2.5$ & $22.9(18.7-29.4)$ \\
FSH/hMG dose $(I U)$ & $2,245 \pm 614$ & $2,145(1,025-3,850)$ \\
Infertility time (yr) & $2.9 \pm 2.3$ & $2(1-10)$ \\
Number of mature oocytes & $5.5 \pm 3.4$ & $5(1-14)$ \\
Number of immature oocytes & $1.5 \pm 1.8$ & $1(0-7)$ \\
Number of immature oocytes in prophase I & $0.8 \pm 1.3$ & $0(0-5)$
\end{tabular}

US, ultrasound; AFC, antral follicle count; FSH, follicle-stimulating hormone; LH, luteinizing hormone; AMH, anti-Müllerian hormone; BMI, body mass index; hMG, human menopausal gonadotropin. 


\section{Obstetrics \& Gynecology Science}

Viviane Margareth Scantamburlo, et al. Ovarian reserve and oocyte quality

hMG dose and number of mature oocytes, Spearman's correlation coefficients were estimated. When evaluating the association between patient- and oocyte-related variables, we considered the hierarchical structure of the data (oocytes within patients) and adjusted the univariate multilevel logistic regression analysis by defining the oocyte as level 1 and the patient as level 2. The significance of variables was assessed using the Wald test, and the measure of association was presented as the odds ratio (OR) with respective 95\% confidence intervals (Cls). Statistical significance was set at $P<0.05$. Data were analyzed using Stata v. 14 software (Stata Corporation, College Station, TX, USA).

\section{Results}

Among 534 patients treated during the study period, 182 were excluded because they were older than 35 years, 259 did not meet the clinical criteria for inclusion, and 44 had incomplete data in their medical records. The main cause of infertility was severe male factor (34.7\%), followed by ovulatory factor (14.3\%). Forty-nine patients were included, and 348 oocyte images were analyzed. The mean patient age was $31.1 \pm 3.3$ years and the mean body mass index was $23.2 \pm 2.5 \mathrm{~kg} / \mathrm{m}^{2}$. Table 2 presents the characteristics of the participants

The distribution into groups according to patient NOR or DOR differed based on the measurements (Fig. 1). Conse-

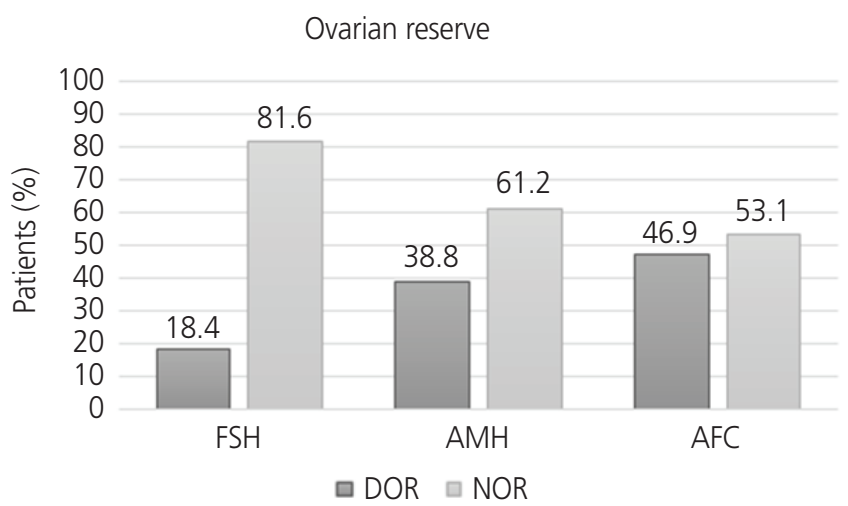

Fig. 1. Classification of patients into groups according to normal or decreased ovarian reserve ${ }^{\text {a) }}$ with respect to basal follicle-stimulating hormone (FSH) levels, anti-Müllerian hormone (AMH) levels, or antral follicle count (AFC). DOR, decreased ovarian reserve;

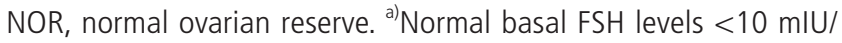
$\mathrm{mL}$, AMH levels $\geq 1.2 \mathrm{ng} / \mathrm{mL}$, and $A F C \geq 10(\mathrm{n}=49)$. quently, the same patient may have been classified as having DOR according to more than one ovarian reserve measurement. When DOR was defined according to basal FSH levels of $\geq 10 \mathrm{mlU} / \mathrm{mL}$, 9 patients (18.4\%) were identified. When DOR was defined as AMH levels $<1.2 \mathrm{ng} / \mathrm{mL}, 19$ patients $(38.8 \%)$ were identified. When DOR was defined as AFC $<10,23$ patients $(46.9 \%)$ were identified.

Patients with DOR defined by AMH levels or AFCs had higher basal FSH levels than those with NOR (Table 3). However, this average was lower than the cutoff point for DOR defined by basal FSH levels ( $\geq 10 \mathrm{mlU} / \mathrm{mL}$ ). No differences related to the total administered dose of FSH/hMG were found when patients were classified according to the measurements for ovarian reserve.

An association was found between AMH levels $<1.2 \mathrm{ng} / \mathrm{mL}$ and the probability of having an immature oocyte (OR, 3.3; $95 \% \mathrm{Cl}, 1.2-8.8 ; P=0.017)$. When the patient's ovarian reserve was defined by basal FSH levels, the estimated OR was $4.4(95 \% \mathrm{Cl}, 1.2-15.5 ; P=0.023)$, indicating that a patient with an FSH level $\geq 10 \mathrm{mlU} / \mathrm{mL}$ had 4.4 times the likelihood of having an immature oocyte than a patient with an $\mathrm{FSH}$ level $<10 \mathrm{mlU} / \mathrm{mL}$. For each additional unit of basal FSH levels, patients had an additional $23 \%$ likelihood of having an immature oocyte $(\mathrm{OR}, 1.23 ; 95 \% \mathrm{Cl}, 1.0-1.5 ; P=0.040)$.

When DOR was defined according to the AFC, an increase in the risk of abnormally shaped oocytes was observed (OR, 2.9; $95 \% \mathrm{Cl}, 1.1-7.6 ; P=0.035)$. When DOR was defined according to $\mathrm{AMH}$ levels, the risk of having oocytes with alterations in the perivitelline space was significantly higher (OR, 3.0; $95 \% \mathrm{Cl}, 1.2-7.6 ; P=0.018)$. Similar results were found

Table 3. Serum basal FSH levels when decreased ovarian reserve was defined according to AMH levels or AFC

\begin{tabular}{cccc}
\hline & $\begin{array}{c}\text { Patients } \\
(\mathbf{n}=\mathbf{4 9})\end{array}$ & $\begin{array}{c}\text { Basal serum FSH } \\
\text { levels }(\mathbf{m l U} / \mathbf{m L})\end{array}$ & P-value $^{\text {a) }}$ \\
\hline AMH $(\mathrm{ng} / \mathrm{mL})$ & & $8.8 \pm 2.4$ & $<0.001$ \\
$<1.2$ & 19 & $6.5 \pm 2.6$ & \\
$\geq 1.2$ & 30 & & 0.021 \\
AFC (n) & & $8.5 \pm 3.3$ & \\
$<10$ & 23 & $6.4 \pm 1.6$ & \\
$\geq 10$ & 26 & & \\
\hline
\end{tabular}

Values are presented as mean \pm standard deviation.

$\mathrm{FSH}$, follicle-stimulating hormone; $\mathrm{AMH}$, anti-Müllerian hormone; AFC, antral follicle count.

a) Non-parametric Mann-Whitney $U$ test. 


\title{
Obstetrics \& Gynecology Science
}

\author{
Vol. 64, No. 6, 2021
}

with respect to the presence of changes in the cytoplasm of the oocyte, which were 5.3 times more likely to be found in patients with DOR than in those with NOR $(\mathrm{OR}, 5.3 ; 95 \% \mathrm{Cl}$, 1.7-16.7; $P=0.004)$.

No significant relationship was observed between the presence of alterations in the zona pellucida and the variables for ovarian reserve or between patient age and the applied oocyte quality parameters. Table 4 shows the association between methods used to measure ovarian reserve, administered dose of gonadotropins in COS, and oocyte quality.

\section{Discussion}

Our results showed that women up to 35 years of age who undergo COS exhibit poor oocyte quality, namely alterations in morphology and immaturity, when they present with DOR.

The higher number of immature and abnormal oocytes in women with DOR according to AMH levels, even if they had normal FSH levels, revealed that FSH alone may not be a good indicator of oocyte quality. Indeed, it has been suggested that basal FSH is a late marker of ovarian reserve, which corroborates the difference observed in our results [2-4].

As a result, $A M H$ and AFC levels are good markers for predicting ovarian response to hormonal stimulation and pregnancy $[3,7]$. The cutoff for DOR is $10 \mathrm{mIU} / \mathrm{mL}$ for FSH levels, $1.25 \mathrm{ng} / \mathrm{mL}$ for $\mathrm{AMH}$ levels, and $10 \mathrm{mlU} / \mathrm{mL}$ for $\mathrm{AFCs}$ [3]. However, there is no consensus in the literature that a correlation exists between these levels and oocyte quality. In our study, using the same cutoff point for basal FSH levels and AFCs but a more stringent cutoff for AMH levels, we found differences in oocyte morphology according to ovarian reserve in young women.

AMH and AFC levels are important predictors for the early identification of DOR before it reaches a critical level $[2,17]$. In our study, the mean basal FSH level for DOR when defined according to $\mathrm{AMH}$ levels was $8.8 \pm 2.4 \mathrm{mlU} / \mathrm{mL}(P<0.001)$. This finding is notable considering that worsening of reproductive outcome is described in the literature when basal FSH levels were $>10 \mathrm{mlU} / \mathrm{mL}[3,18]$. Therefore, our data show that the cutoff for basal FSH levels for defining DOR impacting oocyte alteration could be lower.

Our findings suggest that $\mathrm{AMH}$ is a strong marker of oocyte quality in young women who undergo $\operatorname{COS}$ as changes in the cytoplasm are considered to be the most significant
Table 4. Association between variables of patients and oocyte quality according to maturity and morphology in young women aged up to 35 years who underwent controlled ovarian stimulation $(n=49)$

\begin{tabular}{|c|c|c|}
\hline Variable & OR $(95 \% \mathrm{Cl})$ & $P$-value \\
\hline \multicolumn{3}{|l|}{ Oocyte immaturity } \\
\hline Age & $1.1(0.9-1.3)$ & 0.151 \\
\hline FSH & $1.2(1.1-1.5)$ & 0.040 \\
\hline $\mathrm{AMH}<1.2$ & $3.3(1.2-8.8)$ & 0.017 \\
\hline $\mathrm{AFC}<10$ & $1.2(0.4-3.5)$ & 0.704 \\
\hline $\mathrm{FSH} \geq 10$ & $4.4(1.2-15.5)$ & 0.023 \\
\hline $\mathrm{FSH} / \mathrm{hMG}$ dose $\mathrm{e}^{\mathrm{b})}$ & $0.8(0.5-1.2)$ & 0.329 \\
\hline \multicolumn{3}{|l|}{ Abnormal shape } \\
\hline Age & $1.1(0.9-1.3)$ & 0.356 \\
\hline FSH & $1.1(0.9-1.4)$ & 0.281 \\
\hline $\mathrm{AMH}<1.2$ & $2.3(0.8-6.8)$ & 0.127 \\
\hline $\mathrm{AFC}<10$ & $2.9(1.1-7.6)$ & 0.035 \\
\hline $\mathrm{FSH} \geq 10$ & $1.5(0.3-6.6)$ & 0.625 \\
\hline $\mathrm{FSH} / \mathrm{hMG}$ dose $\mathrm{e}^{\mathrm{b})}$ & $0.9(0.6-1.4)$ & 0.770 \\
\hline \multicolumn{3}{|c|}{ Abnormal zona pellucida } \\
\hline Age & $1.1(0.7-1.6)$ & 0.696 \\
\hline FSH & $1.4(0.8-2.4)$ & 0.282 \\
\hline $\mathrm{AMH}<1.2$ & $2.4(0.2-33.6)$ & 0.517 \\
\hline $\mathrm{AFC}<10$ & $1.3(0.1-16.7)$ & 0.830 \\
\hline $\mathrm{FSH} \geq 10$ & $2.0(0.1-69.2)$ & 0.703 \\
\hline $\mathrm{FSH} / \mathrm{hMG}$ dose $\mathrm{e}^{\mathrm{b})}$ & $2.1(0.7-6.3)$ & 0.193 \\
\hline \multicolumn{3}{|c|}{ Abnormal perivitelline space } \\
\hline Age & $1.1(1.0-1.3)$ & 0.176 \\
\hline FSH & $1.1(0.9-1.3)$ & 0.340 \\
\hline $\mathrm{AMH}<1.2$ & $3.0(1.2-7.6)$ & 0.018 \\
\hline $\mathrm{AFC}<10$ & $1.9(0.8-4.9)$ & 0.160 \\
\hline $\mathrm{FSH} \geq 10$ & $1.1(0.3-4.3)$ & 0.831 \\
\hline $\mathrm{FSH} / \mathrm{hMG}$ dose $\mathrm{e}^{\mathrm{b})}$ & $1.0(0.7-1.4)$ & 0.978 \\
\hline \multicolumn{3}{|l|}{ Abnormal cytoplasm } \\
\hline Age & $1.1(0.9-1.4)$ & 0.182 \\
\hline FSH & $1.4(1.1-1.8)$ & 0.006 \\
\hline $\mathrm{AMH}<1.2$ & $5.3(1.7-16.7)$ & 0.004 \\
\hline $\mathrm{AFC}<10$ & $2.1(0.6-7.1)$ & 0.224 \\
\hline $\mathrm{FSH} \geq 10$ & $3.3(0.6-16.7)$ & 0.150 \\
\hline $\mathrm{FSH} / \mathrm{hMG}$ dose $\mathrm{e}^{\mathrm{b})}$ & $1.5(0.9-2.4)$ & 0.110 \\
\hline
\end{tabular}

Data presented are in $\mathrm{mIU} / \mathrm{mL}$ for basal FSH levels, $\mathrm{ng} / \mathrm{mL}$ for $\mathrm{AMH}$ levels, in years for age, and in IU for FSH/hMG dose.

FSH, follicle-stimulating hormone; $\mathrm{AMH}$, anti-Müllerian hormone; hMG, human menopausal gonadotropin; AFC, antral follicle count; $\mathrm{FSH}$, follicle-stimulating hormone; $\mathrm{OR}$, odds ratio; $\mathrm{Cl}$, confidence interval.

${ }^{a}$ Unnivariate multilevel logistic regression, $P<0.05$; ${ }^{b}$ Each $500 \mathrm{IU}$. 


\section{Obstetrics \& Gynecology Science}

Viviane Margareth Scantamburlo, et al. Ovarian reserve and oocyte quality

factors affecting embryo outcome and implantation potential $[19,20]$. Moreover, minor changes in basal FSH levels negatively affect cytoplasm quality in the oocyte, even in patients considered to have NOR according to this marker. Therefore, in light of the reproductive potential, it would be risky to wait for a woman to demonstrate an abnormal basal FSH level before including her in a group with poor prognosis.

The results of the present analysis are corroborated by those of a previous prospective study that evaluated the rate of aneuploidy in groups defined according to ovarian reserve and included 327 patients who underwent in vitro fertilization and blastocyst biopsy [18]. Patients were considered to have DOR when their basal FSH level was $>10 \mathrm{mlU} / \mathrm{mL}$ and/or their AMH level was $\leq 1.0 \mathrm{ng} / \mathrm{mL}$. As a result, the group with alterations in ovarian reserve tests had a greater proportion of aneuploid embryos (66\% in the group with alterations vs. $51.7 \%$ in the group with normal reserve; $P<0.05)$. The poorest results were found in the group with alterations in $\mathrm{FSH}$ and $\mathrm{AMH}$ levels, with embryonic chromosomal abnormality reaching $77.2 \%$. When alterations were only seen in $\mathrm{AMH}$ levels, the rate of aneuploidy was $58.8 \%$.

Our patients with NOR defined by AFCs had a greater number of mature oocytes $(P<0.001)$. Decreased reserve as defined by this marker indicated a 2.9 times greater risk of having oocytes with an altered shape $(95 \% \mathrm{Cl}, 1.1-7.6$; $P=0.035)$. However, studies have not shown an association between changes in oocyte shape and results of in vitro fertilization, suggesting that this is not an important finding $[10,13,21]$.

Patients with oocytes having normal perivitelline space have been shown to have a higher rate of fertilization ( $60.3 \%$ when normal vs. $37.5 \%$ when increased) and better embryonic development [21]. The patients in our study with DOR defined by $\mathrm{AMH}$ levels showed a stronger association with the occurrence of changes in the perivitelline space, which could lead to poorer reproductive outcomes.

No differences related to the total dose of FSH/hMG administered during ovarian stimulation were observed between any of the groups, and no increased risk of morphological alterations to oocytes was observed when this variable was considered. Therefore, it can be assumed that the quantity of medication administered to patients in this study did not have a significant impact on oocyte quality, which could have affected the analysis.

One limitation of our study was that a critical analysis of intra-observer reliability was not possible for AFCs as this was a retrospective study that only involved collecting data from medical records without the availability of dynamic echographic images, and consequently, there could be a detection bias. In addition, since sperm quality is an important factor for determining fertilization rate and embryonic development and the medical center where the treatments were performed is a reference for treating male-factor infertility, we chose not to assess the oocyte fertilization rate and gestational outcomes. For this reason, further research is needed on couples without associated male-factor infertility. Other limitations of this study were its retrospective design and the small number of patients. Therefore, a prospective study with a larger number of patients is warranted.

This study suggests that age alone does not reflect oocyte quality. Consequently, a simple correlation between age and the success rate of assisted reproductive technology may not be meaningful for patients with DOR up to 35 years of age. Accordingly, for better reproductive planning and to clarify optimal biological time to become pregnant, the ovarian reserve in those at risk of premature ovarian failure (even in young patients) needs to be investigated.

\section{Conflict of interest}

No potential conflict of interest relevant to this article was reported.

\section{Ethical approval}

This study was approval by the Institutional Review Board. The study was performed in accordance with the principles of the Declaration of Helsinki.

\section{Patient consent}

Written informed consent and the use of images from patients are not required for the publication. 


\section{Obstetrics \& Gynecology Science}

Vol. 64, No. 6, 2021

\section{Funding information}

None.

\section{Acknowledgments}

I would like to thank Solena Ziemer Kuzma, who helped with the statistical analysis.

\section{References}

1. American College of Obstetricians and Gynecologists Committee on Gynecologic Practice and Practice Committee. Female age-related fertility decline. Committee Opinion No. 589. Fertil Steril 2014;101:633-4.

2. Wallace WH, Kelsey TW. Human ovarian reserve from conception to the menopause. PLoS One 2010;5:e8772.

3. Practice Committee of the American Society for Reproductive Medicine. Testing and interpreting measures of ovarian reserve: a committee opinion. Fertil Steril 2015;103:e9-17.

4. European Society of Human Reproduction and Embryology. Management of women with premature ovarian insufficiency. Guideline of the european society of human reproduction and embryology [Internet]. Belgium (EU): European Society of Human Reproduction and Embryology; c2015 [cited 2017 Oct 14]. Available from: https://www.eshre.eu/Guidelines-and-Legal/Guidelines/ Management-of-premature-ovarian-insufficiency.aspx.

5. Tarasconi B, Tadros T, Ayoubi JM, Belloc S, de Ziegler $D$, Fanchin R. Serum antimüllerian hormone levels are independently related to miscarriage rates after in vitro fertilization-embryo transfer. Fertil Steril 2017;108:51824.

6. Toner JP, Seifer DB. Why we may abandon basal folliclestimulating hormone testing: a sea change in determining ovarian reserve using antimüllerian hormone. Fertil Steril 2013;99:1825-30.

7. Fleming R, Seifer DB, Frattarelli JL, Ruman J. Assessing ovarian response: antral follicle count versus anti-Müllerian hormone. Reprod Biomed Online 2015;31:486-96.

8. Pils S, Promberger R, Springer S, Joura E, Ott J. Decreased ovarian reserve predicts inexplicability of recur- rent miscarriage? A retrospective analysis. PLoS One 2016;11:e0161606.

9. Demko ZP, Simon AL, McCoy RC, Petrov DA, Rabinowitz $\mathrm{M}$. Effects of maternal age on euploidy rates in a large cohort of embryos analyzed with 24-chromosome single-nucleotide polymorphism-based preimplantation genetic screening. Fertil Steril 2016;105:1307-13.

10. Sunkara SK, Khalaf Y, Maheshwari A, Seed P, Coomarasamy $A$. Association between response to ovarian stimulation and miscarriage following IVF: an analysis of 124351 IVF pregnancies. Hum Reprod 2014;29:1218-24.

11. La Marca A, Minasi MG, Sighinolfi G, Greco P, Argento $C$, Grisendi $V$, et al. Female age, serum antimüllerian hormone level, and number of oocytes affect the rate and number of euploid blastocysts in in vitro fertilization/intracytoplasmic sperm injection cycles. Fertil Steril 2017;108:777-83.e2.

12. Dal Canto M, Guglielmo MC, Mignini Renzini M, Fadini R, Moutier C, Merola M, et al. Dysmorphic patterns are associated with cytoskeletal alterations in human oocytes. Hum Reprod 2017;32:750-7.

13. Setti AS, Figueira RC, Braga DP, Colturato SS, laconelli A Jr, Borges E Jr. Relationship between oocyte abnormal morphology and intracytoplasmic sperm injection outcomes: a meta-analysis. Eur J Obstet Gynecol Reprod Biol 2011;159:364-70.

14. Bosch E, Labarta E, Kolibianakis E, Rosen M, Meldrum D. Regimen of ovarian stimulation affects oocyte and therefore embryo quality. Fertil Steril 2016;105:560-70.

15. Baker VL, Brown MB, Luke B, Smith GW, Ireland JJ. Gonadotropin dose is negatively correlated with live birth rate: analysis of more than 650,000 assisted reproductive technology cycles. Fertil Steril 2015;104:1145-52. e1-5.

16. European Society of Human Reproduction and Embryology. Atlas of human embryology [Internet]. Belgium (EU): European Society of Human Reproduction and Embryology; c2016 [cited 2017 Sep 14]. Available from: http:// eshre.webenpruebas.net.

17. Amanvermez R, Tosun M. An update on ovarian aging and ovarian reserve tests. Int J Fertil Steril 2016;9:411-5.

18. Katz-Jaffe MG, Surrey ES, Minjarez DA, Gustofson RL, Stevens JM, Schoolcraft WB. Association of abnormal ovarian reserve parameters with a higher incidence of aneuploid blastocysts. Obstet Gynecol 2013;121:71-7. 


\section{Obstetrics \& Gynecology Science}

Viviane Margareth Scantamburlo, et al. Ovarian reserve and oocyte quality

19. Balaban B, Urman B. Effect of oocyte morphology on embryo development and implantation. Reprod Biomed Online 2006;12:608-15.

20. Braga DP, Setti AS, Figueira Rde C, Machado RB, laconelli A Jr, Borges E Jr. Influence of oocyte dysmorphisms on blastocyst formation and quality. Fertil Steril
2013; 100:748-54.

21. Xia P. Intracytoplasmatic sperm injection: correlation of oocyte grade based on polar body, perivitelline space and cytoplasmic inclusions with fertilization rate and embryo quality. Human Reprod 1997;12:1750-5. 